\title{
Optical and near-infrared observations of the GRB 970616 error box $^{\star, \star \star}$
}

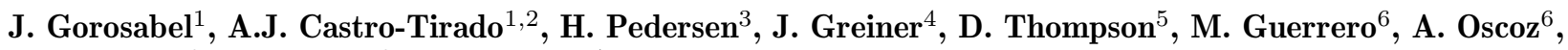 \\ N. Sabalisck ${ }^{6}$, E. Villaver ${ }^{6}$, and N. Lund ${ }^{7}$ \\ 1 Laboratorio de Astrofísica Espacial y Física Fundamental (LAEFF-INTA), P.O. Box 50727, E-28080 Madrid, Spain \\ 2 Instituto de Astrofísica de Andalucía (IAA-CSIC), P.O. Box 03004, E-18080 Granada, Spain \\ 3 Copenhagen University Observatory, Juliane Maries Vej 30 DK 2100 Copenhagen Ø, Denmark \\ 4 Astrophysikalisches Institut Potsdam, D-14482 Potsdam, Germany \\ 5 Max-Planck-Institut für Astronomie, Heidelberg, Germany \\ 6 Instituto de Astrofísica de Canarias, E-38200 La Laguna, Tenerife, Spain \\ 7 Danish Space Research Institute, Juliane Maries Vej 30 DK-2100 Copenhagen $\varnothing$, Denmark
}

Received December 29, 1998; accepted May 18, 1999

\begin{abstract}
We report on near-infrared and optical observations of the GRB 970616 error box and of the X-ray sources discovered by ASCA and ROSAT in the region. No optical transient was found either within the IPN band or in the X-ray error boxes, similarly to other bursts, and we suggest that either considerable intrinsic absorption was present (like GRB 970828) or that the optical transient displayed a very fast decline (like GRB 980326 and GRB 980519).
\end{abstract}

Key words: methods: observational — gamma-rays: bursts

\section{Introduction}

The error box of the BATSE gamma-ray burst GRB 970616 (Connaughton et al. 1997) was scanned by RXTE four hours after the event (Marshall et al. 1997). Observations revealed a previously unknown X-ray source inside the error box whose position was consistent with the one provided later on by the Interplanetary Network (IPN), yielding a combined RXTE/IPN trapezoidal error box (Hurley et al. 1997). Four days later, the ASCA satellite observed the RXTE/IPN error box and detected four X-ray sources. One of them, A\#1 according to the nomenclature of Murakami et al. (1997), was suggested as the X-ray counterpart to GRB 970616. Two candidates were initially proposed by Galama et al. (1997) and Udalski (1997), but other observers (Pahre et al. 1997; Dey et al. 1997; Wheeler et al. 1997; Castro-Tirado et al. 1997) failed to confirm these.

Send offprint requests to: J. Gorosabel (jgu@laeff.esa.es)

* Based on observations collected at the German-Spanish Astronomical Centre, Calar Alto, operated by the Max-PlanckInstitut für Astronomie, Heidelberg, jointly with the Spanish National Commission for Astronomy.

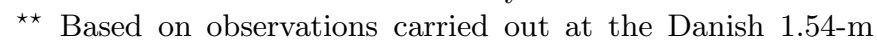
Telescope on the European Southern Observatory, La Silla, Chile.
Observations performed by ROSAT during June 23-25 revealed eleven faint X-ray sources, lying three of them within the RXTE/IPN error box. One of the three sources, R\#2, following Greiner et al. (1997), was consistent with the ASCA variable source A\#1, showing a flux five times lower than the one measured by ASCA. The $3 \sigma$ upper limit to the unabsorbed ROSAT flux of the source $\mathrm{A \# 4}$, implies a factor of seven lower than the previously measured by ASCA. The fading observed during the ASCA observation could suggest that A\#1 is the X-ray counterpart to the GRB. However, the presence of another decaying X-ray source inside the RXTE/IPN error box keeps open the association with the GRB. A massive cluster of galaxies $2^{\prime}$ away from the RXTE/IPN error box is reported by Benítez et al. (1999). Figure 1 shows the locations of the cluster and of the candidates proposed by Galama et al. (1997) and Udalski (1997).

\section{Observations}

\subsection{Near-IR observations}

Observations in the $K^{\prime}$-band were performed with the 3.5-m telescope (+OMEGA) of the Calar Alto Observatory on 1997 June 21.18, 25.17 and 26.18 UT. Also $J$-band images were taken with the $2.2-\mathrm{m}$ Telescope (+MAGIC) on June 25.14. The frames were centered on the candidate proposed by Galama et al. (1997). Additional $K^{\prime}$-band observations were performed on 1998 Oct. 5.125 with the 3.5-m telescope.

\subsection{Optical observations}

Optical observations were mainly taken at La Silla with the Danish 1.54-m telescope equipped with DFOSC, whose field of view $\left(13^{\prime} .6 \times 13^{\prime} .6\right)$ enabled us to cover the four ASCA sources (see Fig. 1). Table 1 displays the observing log. 
Table 1. Observing log of the optical observations performed with the 1.54-m Danish telescope

\begin{tabular}{lccccc}
\hline Date & \multicolumn{5}{c}{ Exposure time $\left(10^{3} \mathrm{~s}\right)$} \\
of 1997 & $B$ & $V$ & $R$ & $i$ & free \\
\hline June 25 & - & - & 8.9 & - & - \\
June 26 & - & - & - & 7.8 & - \\
June 27 & - & - & 9.6 & - & - \\
June 28 & - & - & 10.2 & - & - \\
June 29 & - & - & 11.2 & - & - \\
June 30 & - & 2.0 & 9.0 & - & - \\
July 1 & - & 1.2 & 10.5 & - & - \\
July 2 & 3.6 & - & 7.2 & - & - \\
July 3 & 1.2 & - & - & - & - \\
July 4 & 1.2 & - & - & - & - \\
July 6 & - & - & 3.6 & - & 0.9 \\
July 7 & - & - & - & - & 5.6 \\
\hline Total $26.02^{\mathrm{h}}=$ & 6.0 & +3.2 & +70.2 & +7.8 & +6.5 \\
& & & & & \\
\hline
\end{tabular}

Additional $R$-band imaging was obtained on 1997 July 20.199, 20.207 and 20.257 with the $0.8-\mathrm{m}$ IAC80 telescope at the Observatorio del Teide. A $1020 \times 1024$ pixels CCD provides a $7 ! 4 \times 7 ! 4$ field of view. Limiting magnitude was $R \sim 20$ on the 360 -s exposures.

\section{Discussion and conclusion}

Optical and IR images of the RXTE error box were obtained starting 106 hours after the event. No variable optical counterpart was found within the A\#1 and A \# 4 X-ray source error circles in the RXTE/IPN annulus, being the variation $\Delta B \leq 0.3, \Delta V \leq 0.2, \Delta R \leq 0.2$ and $\Delta i \leq 0.3$ for optical objects with $B \leq 23, V \leq 23, R \leq 24$ and $i \leq 22$. In fact, no objects brighter than $R=24.2$ are seen within the error box of the ROSAT $\mathrm{R} \# 2$ source detected at the ASCA position, consistently with the magnitudes reported by Groot et al. (1997). Therefore, either there is a considerable intrinsic absorption (as in the case of GRB 970828, Yoshida et al. 1999) or the transient optical emission displayed a fast decline, as seen in GRB 980326 (Groot et al. 1998) and GRB 980519 (Hjorth et al. 1999).

No IR variability has been found in a $5^{\prime} \times 4^{\prime}$ region centered at the position of the candidate proposed by Galama et al. (1997). This object was observed at $J=$ $18.3 \pm 0.2$, and $K^{\prime}=17.4 \pm 0.3$ and did not show any obvious IR/optical variation in the $K^{\prime}$-band. The color is consistent with this of a late-type star, as suggested by Pahre et al. (1998) and it seems to be a non-variable star (Groot et al. 1997). Any fading or increase was $\leq 0.5 \mathrm{mag}$ for $K^{\prime} \leq 17.0$.

Concerning the above mentioned cluster of galaxies (the long arrow in Fig. 1), it is probably related to the $\mathrm{X}$-ray source A \# 2, which is outside the IPN band, and consequently not related to the GRB.

Acknowledgements. We wish to thank A. Yoshida for fruitful conversations and the referee N. Masetti for useful suggestions.

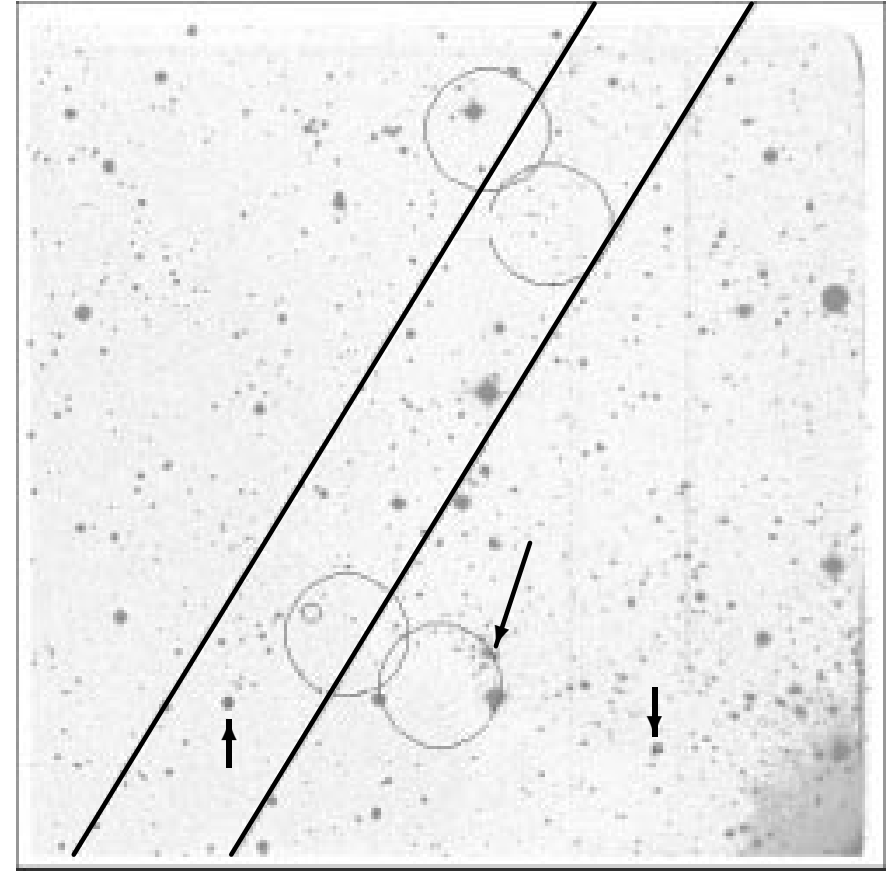

Fig. 1. A coadded $R$-band image of the field of GRB 970616 obtained with the Danish 1.54-m telescope. The total exposure time is 19.5 hours and the limiting magnitude $R=24.2$. The straight thick lines represent the IPN arc determined by Ulysses and BATSE. The two circles fully included in the IPN band are A\#4 (large, at the top) and $R \# 2$ (small at the bottom), whereas the circles partially included are A \# 3 (top) and A \# 1 (bottom). The circle totally outside the IPN band is A \# 2. R\# 1 and $\mathrm{R} \# 3$ are outside the field of view. The two short arrows show the variable objects proposed by Galama et al. (1997) -inside the IPN band- and Udalski (1997) -outside the IPN band-. The field of view is $13.6 \times 13.6$ and is totally within the RXTE/ASM error box. North is to the top and east to the left. The long arrow indicates the massive cluster of galaxies found by Benítez et al. (1999)

This work has been partially supported by Spanish CICYT grant ESP95-0389-C02-02.

\section{References}

Benítez N., et al., 1999 (in preparation)

Castro-Tirado A.J., et al., 1997, IAU Circ. 6688

Connaughton V., et al., 1997, IAU Circ. 6683

Dey A., et al., 1997, IAU Circ. 6696

Galama T., et al., 1997, IAU Circ. 6687

Greiner J., et al., 1997, IAU Circ. 6722

Groot P.J., et al., 1997, IAU Circ. 6723

Groot P.J., et al., 1998, ApJ 502, L123

Hjorth J., et al., 1999, A\&AS (this issue)

Hurley K., et al., IAU Circ. 6687

Marshall F.E., et al., 1997, IAU Circ. 6683

Murakami T., et al., IAU Circ. 6687

Pahre M.A., et al., 1997, IAU Circ. 6691

Udalski A., 1997, IAU Circ. 6690

Wheeler J.C., et al., 1997, IAU Circ. 6697

Yoshida A., et al., 1999, A\&AS (this issue) 